\title{
Global magnetic fields: variation of solar minima
}

\author{
Andrey G. Tlatov ${ }^{1}$ and Vladimir N. Obridko ${ }^{2}$ \\ ${ }^{1}$ Kislovodsk mountian astronomical station of the Pulkovo observatory, \\ Gagarina str. 100, Kislovodsk, Russia \\ email: tlatov@mail.ru \\ ${ }^{2}$ Pushkov Institute of Terrestrial Magnetism, Ionosphere \& Radio Wave Propagation, Russian \\ Academy of Sciences, \\ Troitsk, Moscow Region Russia, \\ email: obridko@mail.ru
}

\begin{abstract}
The topology of the large-scale magnetic field of the Sun and its role in the development of magnetic activity were investigated using $H_{\alpha}$ charts of the Sun in the period 1887-2011. We have considered the indices characterizing the minimum activity epoch, according to the data of large-scale magnetic fields. Such indices include: dipole-octopole index, area and average latitude of the field with dominant polarity in each hemisphere and others. We studied the correlation between these indices and the amplitude of the following sunspot cycle, and the relation between the duration of the cycle of large-scale magnetic fields and the duration of the sunspot cycle.

The comparative analysis of the solar corona during the minimum epochs in activity cycles 12 to 24 shows that the large-scale magnetic field has been slow and steadily changing during the past 130 years. The reasons for the variations in the solar coronal structure and its relation with long-term variations in the geomagnetic indices, solar wind and Gleissberg cycle are discussed.

We also discuss the origin of the large-scale magnetic field. Perhaps the large-scale field leads to the generation of small-scale bipolar ephemeral regions, which in turn support the largescale field. The existence of two dynamos: a dynamo of sunspots and a surface dynamo can explain phenomena such as long periods of sunspot minima, permanent dynamo in stars and the geomagnetic field.
\end{abstract}

Keywords. Solar, large-scale magnetic field, solar activity

\section{Introduction}

The structure of the large-scale magnetic field on the Sun is determined by the distribution of unipolar regions. The pattern of these unipolar regions is evident in the magnetograms and it reflects the results of interaction of all phenomena in the atmospheric layers. Alternatively the unipolar regions can be identified on the $H_{\alpha}$ synoptic charts McIntosh (1979). The prominences and $H_{\alpha}$ filaments, filament channels, $C a I I K$ plages and strong magnetic fields in spots can be used as markers for tracing the evolution and migration of these large-scale magnetic regions. At present, the summary series of $H_{\alpha}$ charts cover the period from 1887 up to now, comparable to the length of sunspot group series (McIntosh 1979, Makarov \& Sivaraman 1989; Vasil'eva 1998), but one can consider the data since 1915 as the most reliable ones. Using filament distribution charts of the Meudon Observatory and daily spectrograms in the $H_{\alpha}$ and $\mathrm{CaIIK}$ lines of the Kodaikanal Observatory (India), the Atlas of $H_{\alpha}$ charts of large-scale magnetic field of the Sun for the period 1915-1964 was constructed (Makarov \& Sivaraman 1989). McIntosh (1979) has constructed $H_{\alpha}$ synoptic charts for the period 1964-1974. Vasil'eva (1998) reconstructed large-scale field topology during 1887-1900 and 1904-1914. There 
are similar data for the period 1975-1991 (SGD, 1975-1991). Beginning from 1979 up to the present time, the bulletin Solnechnye Dannye publishes monthly Synoptic Charts on the basis of the observations at the Kislovodsk Solar Station of the Pulkovo Observatory (S.D.: 1979-2011 http : //www.solarstation.ru). Thus, there is a continuous data stock on the evolution of the large-scale magnetic field for more than 12 cycles of solar activity (Fig. 1). Other data on large-scale magnetic field data come from solar magnetographs. One of the most stable and long-term data series is from Wilcox Solar Observatory. Figure 2 shows a comparison of axisymmetric modes of low harmonics, obtained according to the $H_{\alpha}$ and magnetograph data. You can note the good agreement between the two types of data.

\section{Configuration of Large-Scale Magnetic Fields during the Minimum Activity Epoch}

Long-term variations of large-scale magnetic fields in different latitudes can be studied by means of a series of $H_{\alpha}$ synoptic charts. These charts show boundaries of magnetic field polarities. Routine data obtained at several observatories were used to extract tracers. These are observations of filaments, filament channels, and solar prominences. Thus, in contrast to observations with magnetographs, the spatial resolution of the data is constantly increasing owing to the telescope upgrade, for example. The $H_{\alpha}$ charts contain boundaries on the spherical surface separating positive and negative magnetic polarities. There are various indices for the characterization of large-scale field.

\subsection{Dipole-octopole index of a large-scale magnetic field $\mathbf{A}(\mathbf{t})$}

The photospheric large-scale magnetic field of the Sun can be represented as a function of latitude $\theta$ and longitude $\varphi$, using decomposition in spherical harmonics:

$$
B_{r}=\sum_{l} \sum_{m} P_{l}^{m}\left(g_{l}^{m} \cdot \cos (m \phi)+h_{l}^{m} \cdot \sin (m \phi)\right) \cdot\left[(l+1)\left(R_{\odot} / r\right)^{l+2}+l\left(r / R_{s}\right)^{l-1}\left(R_{\odot} / R_{s}\right)^{l+2}\right]
$$

where $P_{l}^{m}$ - are the Legendre polynomials, $R_{s}$ - radius of the "source surface" (usually $\left.2.5 \cdot R_{\odot}\right)$. The coefficients of decomposition $g_{l}^{m}$ and $h_{l}^{m}$ are surface integrals. Here $B_{r}(\theta, \phi)$ is the value of the surface magnetic field. In the case of $H_{\alpha}$ charts we took only the sign of the magnetic field, keeping the absolute value constant: $+1 \mathrm{G}$ or $-1 \mathrm{G}$, according to whether the field was positive or negative. Nine harmonics were taken into account. It is possible to restore $H_{\alpha}$ charts of a magnetic field using the factors of decomposition $g_{l}^{m}$ and

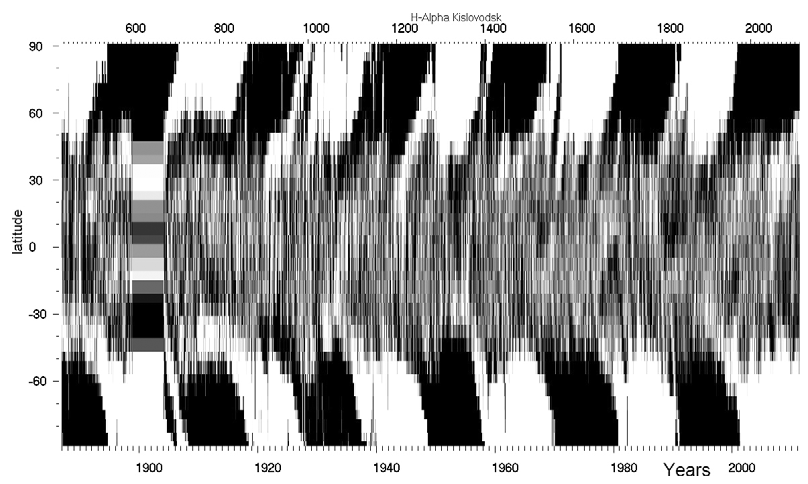

Figure 1. The latitude-time diagram of the polarity distribution of the large-scale magnetic field in the period 1887-2011. 
$h_{l}^{m}$. It was supposed that the magnetic field is a potential field from the photosphere to the "source surface", which was assumed to be situated at $2.5 \cdot R_{\odot}$, where $R_{\odot}$ is the radius of the Sun. Let us consider the time behavior of low- $l$ harmonics. We introduce a parameter $\mathbf{A}(\mathbf{t})$ characterizing the intensity of the dipole and octupole magnetic moments:

$$
\mathbf{A}(\mathbf{t})=\sum_{\mathbf{m}, \mathrm{l}=\mathbf{1}}\left(\mathbf{g}_{\mathrm{l}}^{\mathbf{m}} \mathbf{g}_{\mathrm{l}}^{\mathbf{m}}+\mathbf{h}_{\mathrm{l}}^{\mathbf{m}} \mathbf{h}_{\mathrm{l}}^{\mathbf{m}}\right)+\sum_{\mathbf{m}, \mathbf{l}=\mathbf{3}}\left(\mathbf{g}_{\mathrm{l}}^{\mathbf{m}} \mathbf{g}_{\mathrm{l}}^{\mathbf{m}}+\mathbf{h}_{\mathrm{l}}^{\mathbf{m}} \mathbf{h}_{\mathrm{l}}^{\mathbf{m}}\right) / \mathbf{3}
$$

In Figure 3a (top) the distribution of $\mathbf{A}(\mathbf{t})$ is given for 1915-2011. The 11-yr cycle of a large-scale magnetic field of the Sun leaps to the eye. The procedure of smooth sliding by a window of 2 years was applied to eliminate noise. In Figure 3c (bottom) the solar activity in Wolf numbers, $\mathbf{W}(\mathbf{t})$ is given for comparison.

The $\mathbf{A}(\mathbf{t})$ index demonstrates well-marked 11-year activity cycles, and with respect to the 11-year Wolf number data $\mathbf{W}(\mathbf{t})$ one can see a phase shift in $\mathbf{A}(\mathbf{t})$ of 5 - 6 years. Namely, the $\mathbf{A}(\mathbf{t})$ index cycles precede the sunspot cycles $\mathbf{W}(\mathbf{t})$ (Figure 3a). It should be pointed out that the $\mathbf{A}(\mathbf{t})$ parameter includes only the dipole and octopole components of the background magnetic field, that is, modes $l=1$ and 3. Even-order modes $l=2,4$ and higher-order modes have significantly smaller contributions. Lower and odd-order modes in synoptic charts characterize the configuration of the global solar magnetic fields in the minimum activity epoch.

The maximum value of index $\mathbf{A}(\mathbf{t})$ found near the activity minimum $\left(A_{\max }\right)$ can be used to predict the value of the Wolf number $\mathbf{W}(\mathbf{t})$ at the maximum of the following cycle $\left(W_{\max }\right)$. The correlation coefficient between these parameters is $R=0.96$. The relationship between $A_{\max }$ and $W_{\max }$ can be obtained from the linear regression analysis, and we found $W_{\max }=1320 \cdot A_{\max }-54$. The prediction for the $24 t h$ activity cycle is, therefore, $W_{24}=100 \pm 9$ (Figure $3 \mathrm{a}$ ). The timing when the dipole - octopole index takes the maximum value can slightly vary with regard to the minimum activity epoch. For the prediction of the maximum Wolf number in the $24 t h$ cycle, we adopted the moment when index A takes the maximum value within the current activity minimum. Between the 18 th and the 19th cycles, the maximum of index A took place at the beginning of 1952, which was two years before the Wolf number reached the minimum (Figure 3a).

\subsection{Area of Dominant Polarity at Middle and High Latitudes Apz}

During the minimum activity periods the polar regions are covered with unipolar magnetic fields, and the polarity of the north pole is opposite to that of the south pole. At the same time, the middle and high-latitude zones of each hemisphere are occupied with a field of predominant polarity, which has opposite signs in the two hemispheres. The area covered with the field of dominant polarity can be calculated by using the $H_{\alpha}$ synoptic charts, and can be used as an index (Apz) characterizing the topological organization of large-scale magnetic fields in the polar zones. Figure $3 \mathrm{~b}$ represents the index Apz

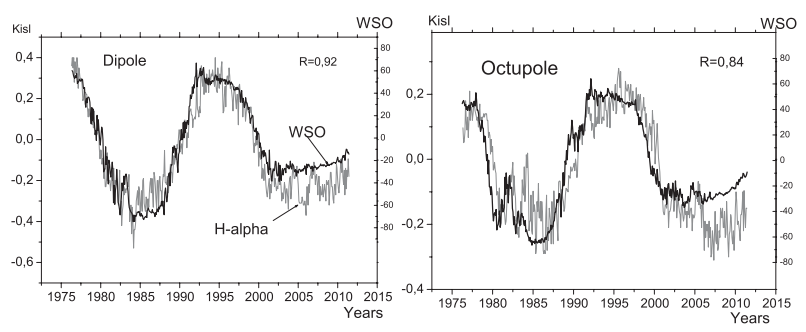

Figure 2. Comparison of axisymmetric modes of low harmonics, obtained according to the $H_{\alpha}$ and magnetograph data. 
calculated for latitudes above $30^{\circ}$ and summed over both hemispheres, expressed in units of $10^{16} \mathrm{~m}^{2}$. Local maxima in the $\mathbf{A p z}$ index are generally found at the minimum activity epochs. An exception is the maximum in Apz between cycles 18 and 19, which took place earlier than the sunspot number minimum. The relationship between the cycle amplitudes in Apz and Wolf numbers can be presented as $W_{\max }=3.92 \cdot A p z_{\max }-364.3,(R=0.91)$. According to the value of $\mathbf{A p z}$ in the current minimum, the amplitude of the 24th cycle can be predicted as $W_{24}=123 \pm 15$ (Figure $3 \mathrm{~b}$ ). This value derived from the linear regression using the data over eight activity cycles is close to the activity amplitude of the $23 r d$ cycle. At the same time, the value of the Apz index itself in the 24th cycle is lower than in the minimum of the $23 \mathrm{rd}$ cycle.

\subsection{Average Latitude of Large-Scale Magnetic Fields}

The average latitude of large-scale magnetic fields of either positive or negative polarity can be considered as another characteristic, revealing the role of the topology of largescale magnetic fields. The parameter may be represented by $\overline{\theta^{p}}=\int_{-\pi}^{\pi} \theta^{p} d s^{p}$, where $\theta^{p}$ is the latitude of a small area $d s^{p}, S p$ is the total area, and index $p$ specifies either positive $(+)$ or negative $(-)$ polarity. Generally the average latitudes of large-scale magnetic fields migrate equatorward. The separation of the opposite polarities $\theta_{ \pm}=a b s\left(\theta_{p}-\theta_{n}\right)$ in the minimum activity epochs is in the range of $25^{\circ}-40^{\circ}$. During the years $1915-2008$, this value was largest between cycles 18 and 19 and smallest between cycles 19 and 20 (Figure $3 \mathrm{c})$. The relationship between the value of $\theta_{ \pm}$in the activity minimum and the amplitude of the following sunspot cycle $\left(W_{\max }\right)$ is found as $W_{\max }=7.7 \cdot \theta_{ \pm}-111,(R=0.89)$. The amplitude of the $24 t h$ cycle predicted from this relationship is $W_{24}=120 \pm 17$ (Figure 3c).

\section{Duration of Cycles Seen in Large-Scale Magnetic Fields and the Prediction of Activity Cycles}

According to the hypothesis of the extended activity cycle (Wilson et al. 1988), the duration of an activity cycle is longer than of the sunspot cycle. Namely, the cycle may

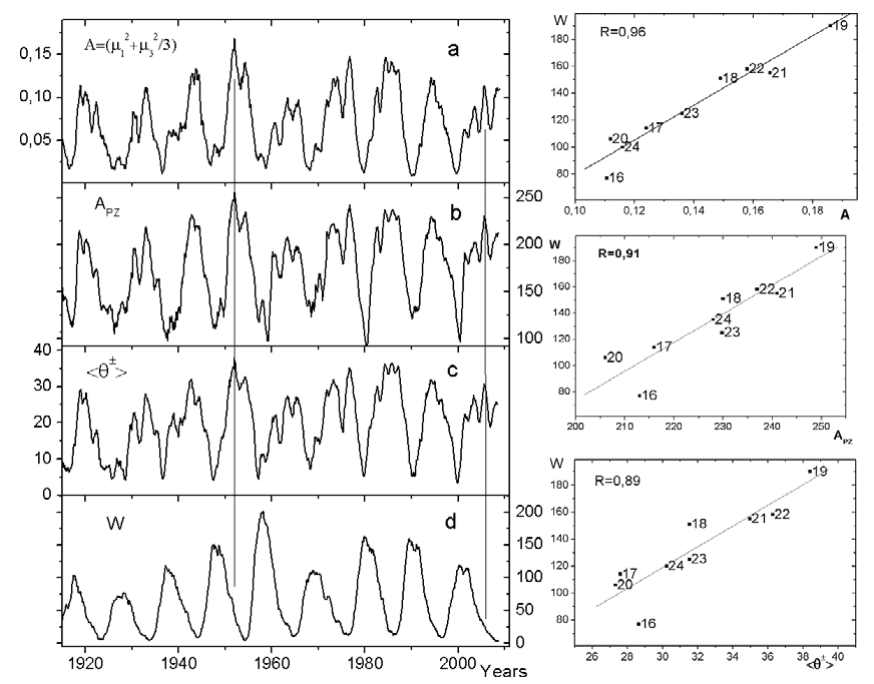

Figure 3. Indices of large-scale magnetic fields: (a) index $\mathbf{A}$, representing the dipole and octopole components, (b) the area of polar zones Apz in latitudes above $30^{\circ}$, in units of $10^{16} \mathrm{~m}^{2}$, the separation between the average latitudes of positive and negative large-scale fields, $\theta_{ \pm}$, expressed in degrees. The Wolf sunspot number $\mathrm{W}$ in panel (d) for comparison. 
begin 1 - 3 years before the approach of sunspot minimum (Harvey 1992). The question is still unresolved, however, when a new activity cycle begins. Let us consider the time interval between the polarity reversal of global magnetic fields derived from the $H_{\alpha}$ synoptic charts and the following sunspot minimum. The moments of polarity reversal $T_{r}^{n}$ is taken from the papers of several authors (Makarov et al. 2003; Tlatov 2009). In case the polarity reversal was not simultaneous on the two hemispheres, we chose the reversal of the hemisphere that took place later. There is a relation between the amplitude of the following sunspot cycle $W_{n+1}$ as a function of the time interval between the polarity reversal of the large-scale magnetic fields and the sunspot cycle minimum, $\Delta T_{R}=T_{\min }^{n+1}-T_{r}^{n}$. The shorter the time interval between the polarity reversal and the beginning of the next sunspot cycle is, the higher is the amplitude of the following sunspot cycle (Tlatov 2009):

$$
W_{\max }^{n+1}=320( \pm 51)-38.2( \pm 9.6) \cdot \Delta T_{R}, R=0.78
$$

There is also a relation between the amplitude of the new sunspot cycle $W_{\max }^{n+1}$ and the time interval $\Delta T_{2}=T_{\max }^{n+1}-T_{r}^{n}$. Here $T_{\max }^{n+1}$ is the time of the new sunspot maximum and $T_{r}^{n}$ is the time of the polarity reversal in the previous cycle. The relationship can be represented as:

$$
W_{\max }^{n+1}=352( \pm 40)-24.9( \pm 4.3) \cdot \Delta T_{2}, \quad R=0.87
$$

The relations between the amplitude of the following sunspot cycle and the time intervals given in Equations (3.1) and (3.2) contain information on the moments of activity minimum and maximum of the following cycle. On the other hand, another relationship given below (Equation (3.3)) involves the parameters available in the current cycle on the right-hand side:

$$
W_{\max }^{n+1}=83( \pm 11)-0.09( \pm 0.02) \cdot W_{\max }^{n+1} \cdot \Delta T_{3} \cdot a b s\left(\Delta T_{3}\right), \Delta T_{3}=T_{\max }^{n}-T_{r}^{n}, R=0.86,
$$

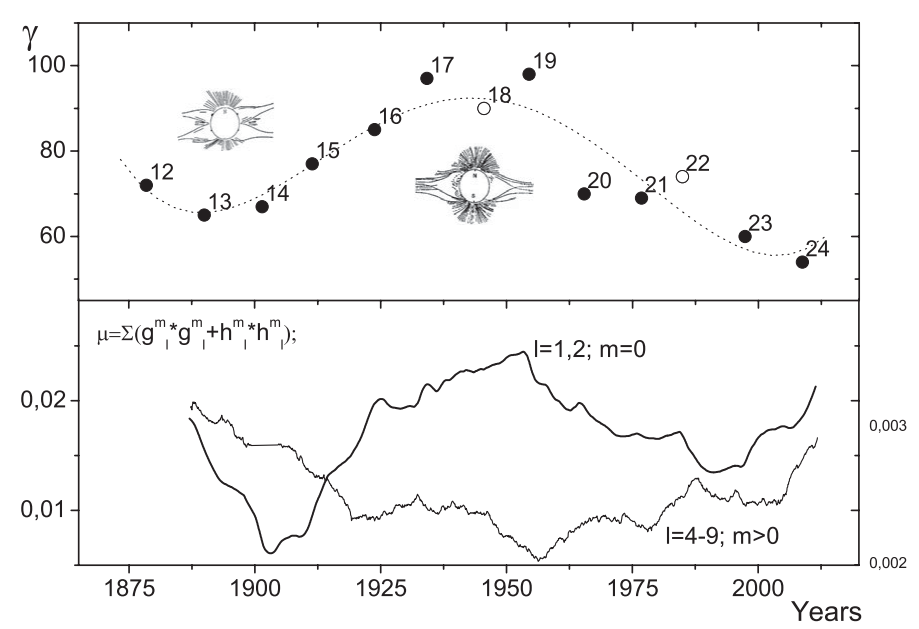

Figure 4. (Upper panel) Distribution of parameter $\gamma$ for the structure of the corona of the minimal type. Solar activity cycles numbers are given. The shapes of the solar corona in cycles 13, 19 are shown. (Lower panel) The amplitudes of the harmonics were smoothed with a "running window" 11 years long. At the bottom of this panel we give the amplitudes of the axisymmetric modes dipole and quadruple $(l=1,2 ; m=0)$ and for harmonics, characterizing the sector structure of the higher harmonics $(l>3 ; m>0)$. 
We can demonstrate a number of other relationships between the time intervals which include the moments of polarity reversal of the large-scale magnetic fields:

$$
\begin{gathered}
W_{\max }^{n+1}=450( \pm 101)-20.7( \pm 6.4) \cdot W_{\max }^{n+1} \cdot \Delta T_{4}, \Delta T_{4}=T_{\max }^{n+1}-T_{r}^{n}, R=0.72, \\
\Delta T_{2}=1.12( \pm 1.3)+1.54( \pm 0,24) \cdot \Delta T_{R}, R=0.88,
\end{gathered}
$$

Earlier in the article by Makarov et al. (2003), the following relation has been found:

$$
T_{r}^{n+1}-T_{r}^{n}=5.8( \pm 0.4)+304( \pm 33) / W_{\max }^{n+1}, R=0.95
$$

Equations (1)-(6) allow us to derive the recurrent formulae for the times $T_{\max }^{n+1}, T_{\text {rev }}^{n}, T_{\min }^{n+1}$ and the amplitude $W_{\max }^{n+1}$ in terms of the parameters $T_{r e v}^{n+1}, T_{\min }^{n}, T_{\max }^{n}, W_{\max }^{n}$. However, due to low correlation coefficients found, their use for the prediction for the forthcoming cycle is not promising. If we assume that the minimum of the 24 th activity cycle took place at $T_{\min }^{24}=2009$ and $T_{r}^{23}=2001.7$, then the amplitude of this cycle can be evaluated as $W^{24}=42 \pm 27$. Equation (3.2) then gives the timing of the maximum of the new activity cycle. If we assume the amplitude of the $24 t h$ cycle as $W^{24}=80$, the maximum of the 24th cycle is expected at $T_{\max }^{24}=2012.6 \pm 0.7$. Equation (3.3) then gives the prediction $W^{24}=92 \pm 21$.

\section{Long-term Changes of Coronal Shape and Geomagnetic Disturbance}

The presence of long-term trends in the solar corona structure can be caused by changes in the configuration of the global magnetic field of the Sun. The role of active region formation during a solar activity minimum is significant. It has long been known that large coronal streamers typically lie above the polarity-inversion lines of the large-scale magnetic field marked by filaments and prominences (Vsekhsvyatskiy et al. 1965). For

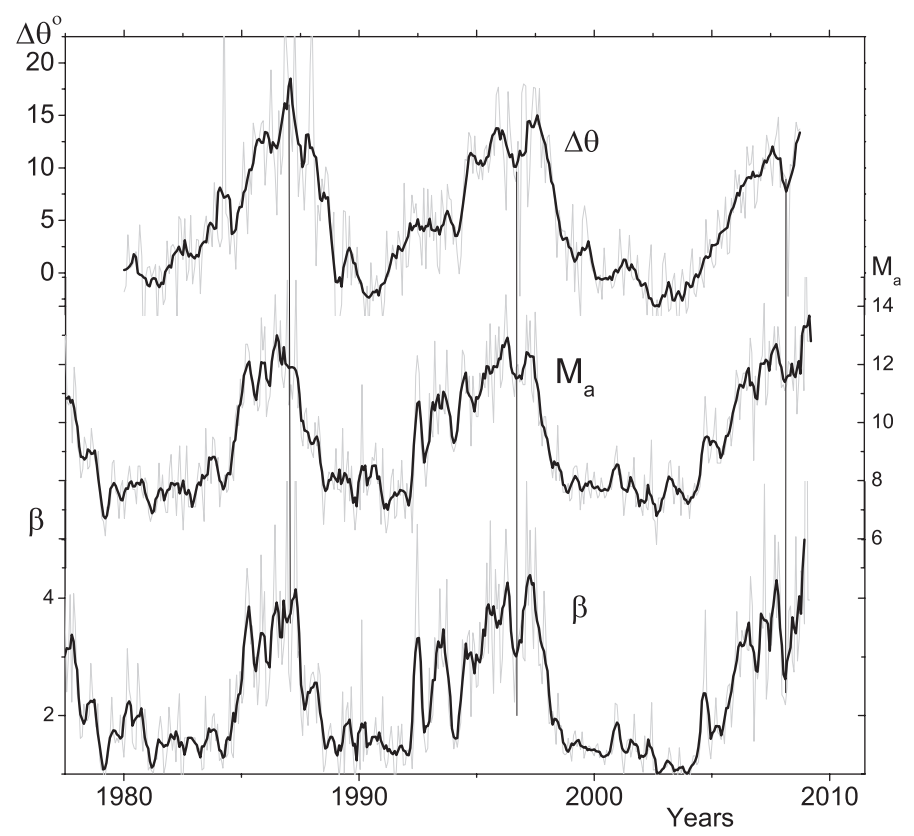

Figure 5. The comparison between a) the deviations of coronal rays $\Delta \theta$ with the solar wind parameters according to OMNI2 database. b) Alfven mach number $M_{a}$. c) Plasma beta $\beta$. Data of the solar wind parameters are smoothed in 20 Bartels rotations. 
this reason, investigations of the large-scale corona shape give valuable information on the structure of the large-scale fields during a long time interval. During the activity minimum, the properties of the global magnetic field of the Sun manifest themselves in the most pronounced way. The magnetic field of the Sun is determined by large-scale structures. The northern and southern hemispheres of the Sun have magnetic fields of opposite polarity. The strength of the polar magnetic field is significantly higher than the fields in middle and low latitudes in the activity minimum period.

To analyze the corona shape at the eclipses during a solar activity minimum epoch in activity cycles 12 to 24 , a corresponding index should be chosen. We need the index that characterizes the shape of the corona of the minimal type and is applicable to images and drawings of different qualities. The corona of the solar minimum is characterized by pronounced polar ray structures and large coronal streamers (Pasachoff et al. 2008). Let us introduce the parameter $\gamma$ that characterizes the angle between high-latitude boundaries of the large coronal streamers at a distance of $2 \cdot R_{\odot}$. The $\gamma$ parameter is the sum of the angles at the eastern and western limbs: $\gamma=180-\left(\gamma_{W}+\gamma_{E}\right)$. A parameter $\gamma$ can be introduced for these eclipses. This parameter reduces parameter $\gamma$ to the minimum phase. The application of this procedure is effective for recognizing the shape of the corona close to the minimum activity with the phase $\Phi<0.4$. Figure 4 presents variations in parameter $\gamma$ during the last 13 activity cycles.

The link between the coronal shape, geomagnetic disturbances and solar wind parameters can be clearly seen in 11-year activity cycle. Global magnetic field can lead to non-radial spreading of the coronal streamers and probably, to the spreading of the solar wind. In minimal activity when the value of the global field reaches its maximum, coronal rays are deflected aside from the helio-equator.

The solar corona structure corresponds to the configuration of solar magnetic fields. Since the magnetic field of the Sun is subjected to cyclic variations, the corona shape also changes cyclically. The coronal rays are distinctive structures in the solar corona, which propagate at a small angle to the radial direction from the Sun and display the electron density in K-corona enhanced by the factor of 3 to 10 . The angle $\Delta \theta$ that describes the deviation of the rays from the radial position varies with the phase of the solar cycle and the latitude (Eselevich \& Eselevich 2002; Tlatov 2010).

The regular observations with the SOHO/LASCO and Mark-III/IV coronagraphs at the Mauna Loa solar observatory make it possible to analyze the structure of the solar

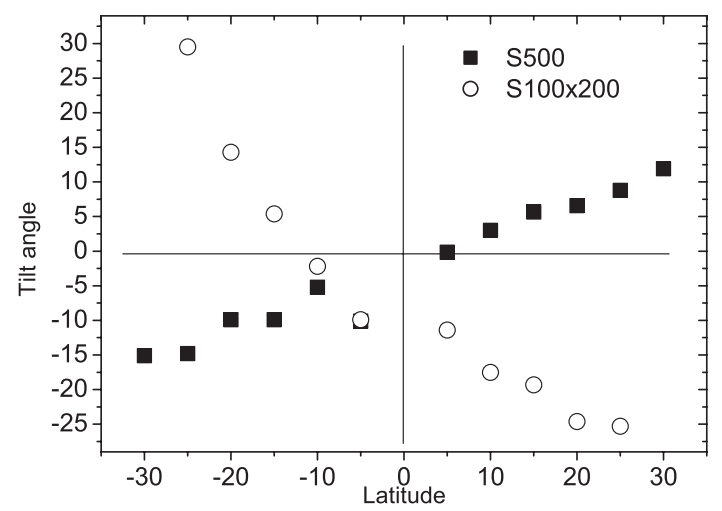

Figure 6. Latitudinal variation of the average tilt of small ephemeral regions (filled squares) and active regions (open circles). In both hemispheres, the following polarity in ERs is situated at lower latitude as compared with the leading polarity. This orientation is opposite to the tilt of ARS Tlatov (2010). 
corona for the time comparable with the duration of the solar cycle. These data substantially complement extended series of observations of the corona in spectral lines carried out with extra-eclipse coronagraphs, since they make it possible to analyze coronal structures at sufficiently large distances from the solar limb, and also occasional observations of the "white" light corona during total eclipses. The coronagraph - polarimeter Mark-III detected the structure of the solar corona at the heights $\sim 1.15: 2.45 R_{\odot}$ in 1980-1999. In 1998, at Mauna Loa observatory the new low-noise coronagraph Mark-IV, with a liquid-crystal modulator of polarization and a CCD, was mounted. To decrease the radial gradient and consequently to increase the contrast, we applied to the Mark data an artificial vignetting function. The LASCO-2 coronagraph telescope on board of SOHO satellite has been working since 1996 and covers the distance $1.5 R_{\odot}: 6 R_{\odot}$ above the solar limb. Thereby, here we have analyzed the structure of the corona for 1980-2008 on the basis of the data obtained at ground-based observations with Mark-III/IV coronagraphs and for 1996-2009 with the SOHO/LASCO-2 data.

In order to determine the deviation of coronal rays, we developed a technique of the identification of coronal streamers in two-dimensional images of the corona obtained with SOHO/LASCO-2 and Mark-III/IV in automatic mode. The analysis is based on discrimination of central parts of bright coronal structures propagating, as a rule, at some angle to the radial direction, discrimination of the points of the local maxima, and determination of the parameters of the approximating line section. Figure 5a presents the behavior of the shape of the coronal rays. But is it possible to establish the link directly between the coronal shape and solar wind parameters, measured from the Earth? Data bases OMNI1 and OMNI2 (http://nssdc.gfc.nasa.gov/omniweb) contain the information concerning hourly average value of key parameters of the solar activity, interplanetary atmosphere and geomagnetic disturbance since 1964 . We collated changes of $\Delta \theta$ parameter with solar wind parameter and magnetic Mach number (Fig. 5). In minimal activity epoch there is a correspondence between the non-radial parameter $\Delta \theta$ and solar wind parameters. Comparing parameters $\beta=8 \pi n k T / B^{2}$ and $M_{a}=v / v_{a}$ we can make a conclusion that non-radial corona influences the relation of the solar wind, which grows in minimum solar activity and therefore magnetic field in minimal activity squeezes the solar wind flux towards helio-equator. These results complement the results of comparison of the three activity minima (Jian et al. 2011). This solar minimum has the slowest, least dense, and coolest solar wind, and the weakest magnetic field.

\section{The fine structure of large-scale solar magnetic field}

In the work by Tlatov et al. (2010) it was established that the orientation of the magnetic axis of ephemeral areas (ER) depends on the character of large-scale field (Fig. 6). However, large-scale magnetic field has zonation of polarity, as it is shown on Fig 8. We applied distribution of directions of magnetic axis ER onto zonality. There is a correspondence of distributions. It means that small-scale dipoles are oriented in
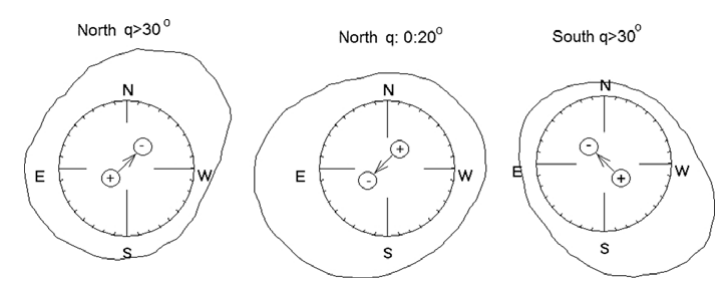

Figure 7. The distribution of the magnetic bipolar axis in ephemeral regions in different zones of the large-scale magnetic field. 
accordance with the large-scale magnetic field distribution. Number of ER is equal to several hundreds, and consequently, ER can influence the formation of the large-scale magnetic field (Fig 7). The diagram shows that dipoles ER can strengthen large-scale magnetic field in near-surface dynamo process (Fig. 8). In the absence of sunspots the presence of ER would result in monotonous growth of the global magnetic field. During regular 11-year cycles the emergence of the sunspots lead to the reversal of the large scale field. But during the grand minima such as Maunder minimum other type of dynamo is possible. In contrast to traditional tachocline the superficial dynamo ER is present. As the result of ER dynamo action the global field is regenerated and the tachocline dynamo of the sunspots is turned over. It is possible that alternation of the activity cycles with the periods of deep minimum of the sunspots, such as Maunder minimum, is a consequence of the predominance of one or another type of dynamo on the Sun.

Constant magnetic activity of some types of stars Hall \& Lockwood (2004) can be accounted for the same mechanism. It is obvious that in this case surface dynamo is prevailing, in some separate cases it can lead to the appearance of the gigantic spots on the poles.

In the Earth's dynamo surface type is also prevailing, and changes of the character is analogous to the sunspots cycles, and takes place periodically.

So, we can conclude that the large-scale field plays the key role in solar magnetism. This follows from the connection between the field configuration and solar activity cycles. Large-scale field, apparently, can exist even without spots due to generation of ephemeral areas. During the periods of spots' absence it can strengthen because of surface dynamo, which leads to the growth of the global magnetic field. Two-component dynamo can account for different phenomena, observed within the sun and stars, and effects of geomagnetism as well.

\section{Overview}

The indices presented above characterize the state of the solar atmosphere in the descending phase and at the minimum activity epoch. Their amplitude is related to the amplitude of the following cycle of activity. Some of these indices are related to the large-scale magnetic fields, and others characterize activity at high latitudes. Before a high cycle of

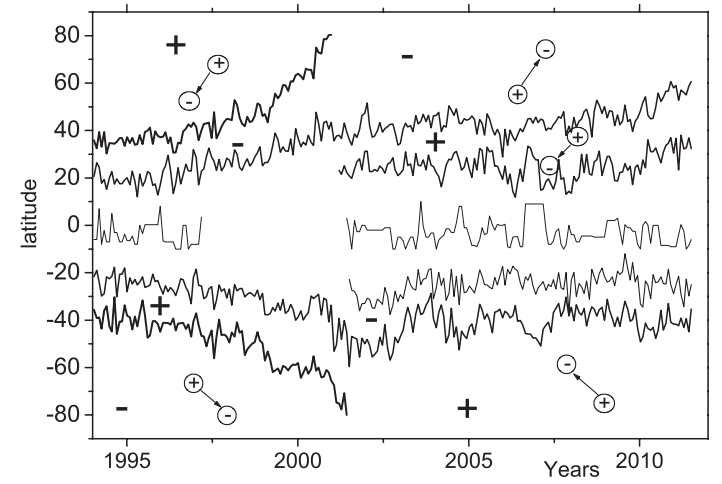

Figure 8. Zonal structure of the large-scale magnetic field according to $H_{\alpha}$ synoptic charts. Solid lines represent migration trajectories of magnetic neutral lines (or the zone boundaries with the opposite polarities on either of their sides in the order $+/-$ or $-/+$ ) of the large-scale magnetic field derived from $H_{\alpha}$ synoptic charts in the northern and southern hemispheres for the period 1994-2011. In the zones of different polarity of the magnetic field are shown the predominant direction of the magnetic axis of ER. 
activity, in each hemisphere of the Sun a part of the large-scale field of prevailing polarity increases (index Apz), and the separation between the average latitudes of the magnetic fields of opposite polarities (in the opposite hemispheres) takes a maximum value (index $\left.\theta_{ \pm}\right)$. These physical conditions provide high values of the dipole-octopole index $\mathbf{A}$. On a unipolar background magnetic field arise polar faculae and calcium bright points.

An important parameter with promising prediction capability is the time interval between the polarity reversal of the large-scale magnetic fields and the sunspot cycle minimum, $\Delta T_{R}=T_{m i n}^{n+1}-T_{r}^{n}$. The smaller this time interval is, the more powerful the sunspots cycle can be expected. Probably, $\Delta T_{R}$ may be interpreted as a latent period of activity cycle development. As we have seen so far, the solar activity cycle can be interpreted as a process beginning with the polarity reversal of the large-scale magnetic fields. The total duration of the solar cycle was found to be 14.3 years for the 18th cycle and 18.6 years for the 14 th cycle.

The analysis of the corona shape has revealed a long-term modulation of the global magnetic field of the Sun. Possibly, a secular modulation exists of the global solar magnetic field that is most pronounced during the solar activity minimum epoch. During the secular cycle of the global magnetic field of the Sun, the relation between the axisymmetric low order components and sectorial components of the magnetic field changes. The largest amplitude of the dipole component occurred during the interval 1944-1955. At the turn of the 19th to 20st and 20th to 21st centuries the solar corona shape and, possibly, the global magnetic field correspond to the configuration close to the octopole one (see Fig. 4).

Perhaps the large-scale field leads to the generation of small-scale bipolar ephemeral regions (Figs. 6, 7, 8), which in turn support the large-scale field. The existence of two dynamos: the dynamo of sunspots and the surface dynamo can explain phenomena such as long periods of sunspot minima, permanent dynamo in stars and the geomagnetic field.

\section{Acknowledgements}

This paper was supported by the Russian Fund of Basic Researches, and Program of the Russian Academy of Science.

\section{References}

Eselevich, V. G. \& Eselevich, M. V. 2002, Solar.Phys., 208, 5

Hall, J. C. \& Lockwood, G. W. 2004, ApJ, 614, 942

Harvey, K. L. 1992, In: Harvey, K. L. (ed.), The Solar Cycle, ASP Conf. Ser. 27, 335

McIntosh, P. S. 1979, Annotated Atlas of $H_{\alpha}$ Synoptic Charts, NOAA, Boulder

Makarov, V. I. \& Sivaraman, K. R. 1989, Solar. Phys., 119, 35

Makarov, V. I., Tlatov, A. G., \& Sivaraman, K. R. 2003, Solar. Phys., 214, 41

Jian, L. K., Russell, C. T., \& Luhmann, J. G. 2011, Solar. Phys., Doi 10.1007/s11207-011-9737-2

Pasachoff, J. M., Rušin, V., Druckmüller, M., Druckmülerová, H., Bělik, M., Saniga, M., Minarovjech, M., Marková, E., Babcock, B. A., Souza, S. P. \& Levitt, J. S. 2008 , ApJ, 682,638

Tlatov, A. G. 2009, Solar. Phys., 260, 465

Tlatov, A. G., Vasil'eva, V. V., \& Pevtsov, A. A. 2010, ApJ, 717, 357

Tlatov, A. G. 2010, ApJ, 714, 805

Vasil'eva, V. V. 1998, in: V. I. Makarov \& A. V. Stepanov (eds.), New Cycle of Activity of the Sun : Observational and Theoretical Aspects (St.-Petersburg, Pulkovo), p. 213

Vsekhsvyatskiy, S. K., Nikolskiy, G. M., Ivanchuk, V. I., \& Nesmyanovich, A. T. et al. 1965, Solar Corona and Corpuscular Radiation in the Interplanetary Space, (Kiev, Naukova Dumka), 293

Wilson, P. R., Altrock, R. C., Harvey, K. L., Martin, S. F., \& Snodgrass, H. B. 1988, Nature 333,748 